Article

\title{
Spectral Function of a Boson Ladder in an Artificial Gauge Field
}

\author{
Roberta Citro ${ }^{1,2, *(\mathbb{D}}$, Stefania De Palo ${ }^{3,4}\left(\mathbb{D}\right.$, Nicolas Victorin $^{5}$, Anna Minguzzi ${ }^{5}(\mathbb{D})$ \\ and Edmond Orignac ${ }^{6}$ (D) \\ 1 Dipartimento di Fisica “E.R. Caianiello", Università degli Studi di Salerno and CNR-Spin, Via Giovanni Paolo II, \\ 132, I-84084 Fisciano (Sa), Italy \\ 2 INFN, Sezione di Napoli, 80126 Napoli NA, Italy \\ 3 CNR-IOM-Democritos National Simulation Centre, UDS Via Bonomea 265, I-34136 Trieste, Italy; \\ stefaniadepalo@gmail.com \\ 4 Dipartimento di Fisica Teorica, Università di Trieste, I-34136 Trieste, Italy \\ 5 Univ Grenoble Alpes, CNRS, LPMMC, 38000 Grenoble, France; nicolas.victorin@lpmmc.cnrs.fr (N.V.); \\ anna.minguzzi@lpmmc.cnrs.fr (A.M.) \\ 6 Univ Lyon, Ens de Lyon, Univ Claude Bernard, CNRS, Laboratoire de Physique, F-69342 Lyon, France; \\ Edmond.Orignac@ens-lyon.fr \\ * Correspondence: rocitro@unisa.it
}

Received: 31 January 2020; Accepted: 8 March 2020; Published: 10 March 2020

\begin{abstract}
We calculate the spectral function of a boson ladder in an artificial magnetic field by means of analytic approaches based on bosonization and Bogoliubov theory. We discuss the evolution of the spectral function at increasing effective magnetic flux, from the Meissner to the Vortex phase, focussing on the effects of incommensurations in momentum space. At low flux, in the Meissner phase, the spectral function displays both a gapless branch and a gapped one, while at higher flux, in the Vortex phase, the spectral function displays two gapless branches and the spectral weight is shifted at a wavevector associated to the underlying vortex spatial structure, which can indicate a supersolid-like behavior. While the Bogoliubov theory, valid at weak interactions, predicts sharp delta-like features in the spectral function, at stronger interactions we find power-law broadening of the spectral functions due to quantum fluctuations as well as additional spectral weight at higher momenta due to backscattering and incommensuration effects. These features could be accessed in ultracold atom experiments using radio-frequency spectroscopy techniques.
\end{abstract}

Keywords: bosonization; Bogoliubov approximation; artificial gauge field; spectral functions

\section{Introduction}

In quasi-one-dimensional systems, analogs of the Meissner and Vortex phase have been predicted for the bosonic two-leg ladder [1-5], the simplest system where orbital magnetic field effects are allowed. It was shown that in this model, the quantum phase transition between the Meissner and the Vortex phase is a commensurate-incommensurate transition [6-8]. Recently, the advent of ultracold atomic gases has opened a route to realize low dimensional strongly interacting bosonic systems [9-11] where an artificial magnetic flux acting on the ladder can be simulated either using geometric phases [12] or the spin-orbit coupling [13,14]. Indeed, there is a mapping of the two-leg ladder bosonic model to a two-component spinor boson model in which the bosons in the upper leg become spin-up bosons, and the bosons in the 
lower leg, spin-down bosons. Under such mapping, the magnetic flux of the ladder becomes a spin-orbit coupling for the spinor bosons. Theoretical proposals to realize either artificial gauge fields and artificial spin-orbit coupling have been put forward $[15,16]$, and an artificial spin-orbit coupling has been achieved in a cold atoms experiment [17]. In a two-leg boson ladder, the transition between a commensurate to an incommensurate phase has been characterized using equal time correlation functions [3,18-20]. However, we expect a direct signature of the transition also in dynamical correlation functions. In one dimension, the low energy modes are collective excitations [21,22], and in the two-leg ladder, there is a separation between a total density ("charge") and a density difference ("spin") mode [2,4]. This is analogous to the well-known spin-charge separation in electronic systems [21] and two-component boson systems [23]. Except at commensurate filling [24-27], the "charge" mode is gapless. By contrast, the "spin" mode is gapped in the Meissner phase and gapless in the Vortex phase, the transition as a function of flux being in the commensurate-incommensurate class [6,7]. Thus, the two phases are characterized by very different dynamical correlation functions. Among those correlation functions, one could, for example, consider the "spin-spin" dynamical structure factor. This would display a well defined gapped or gapless dispersion, respectively, in the Meissner and in the Vortex phase. However, such correlation function would not be sensitive to the incommensuration in the weak interchain hopping regime, although it displays incommensuration features at weak interactions and large interchain hopping $[4,28]$. A better indicator of incommensuration in all regimes is provided by the spectral function of the bosonic particles. In the Vortex phase, it always displays a shift in the position of the minimum of the dispersion away from $q=0$ as a consequence of the incommensuration, whereas in the Meissner state, the minimum of the dispersion remains at $q=0$. A particular feature of the single-particle spectral function is that it is incoherent [23,29], i.e., the low energy excitation branches emerge as power-law singularities instead of delta function singularities. From the experimental point of view, single-particle spectral functions are accessible via radiofrequency (RF) spectroscopy techniques [30-32]. In the present paper, we calculate the boson spectral function in the different phases of the boson ladder at incommensurate filling in order to fully characterize the transition under flux.

\section{Model}

In the following, we use the notations and definitions of [20]. We consider a model of bosons on a two-leg ladder in the presence of an artificial $\mathrm{U}(1)$ gauge field [14,33]:

$$
H=-t \sum_{j, \sigma}\left(b_{j, \sigma}^{\dagger} e^{i \lambda \sigma} b_{j+1, \sigma}+b_{j+1, \sigma}^{\dagger} e^{-i \lambda \sigma} b_{j, \sigma}\right)+\frac{U}{2} \sum_{j, \sigma} n_{j \sigma}\left(n_{j \sigma}-1\right)+\frac{\Omega}{2} \sum_{j, \alpha, \beta} b_{j, \alpha}^{\dagger}\left(\sigma^{x}\right)_{\alpha \beta} b_{j, \beta} .
$$

where $\sigma=\uparrow, \downarrow$ represents the leg index or the internal mode index [34-36], $b_{j, \sigma}$ annihilates a boson on leg $\sigma$ on the $j$-th site, $n_{j \alpha}=b_{j \alpha}^{\dagger} b_{j \alpha}, t$ is the hopping amplitude along the chain, $\Omega$ is the tunneling between the legs or laser-induced tunneling between internal modes, $\lambda$ is the flux of the effective magnetic field, $U$ is the repulsion between bosons on the same leg. The low-energy effective theory for the Hamiltonian (1), where $\Omega \ll t$ is treated as a perturbation, is obtained by using Haldane's bosonization [37]. By introducing the fields $\phi_{\alpha}(x)$ and $\Pi_{\alpha}(x)$ satisfying canonical commutation relations $\left[\phi_{\alpha}(x), \Pi_{\beta}(y)\right]=i \delta(x-y)$ as well as the dual $\theta_{\alpha}(x)=\pi \int^{x} d y \Pi_{\alpha}(y)$ of $\phi_{\alpha}(x)$, and after introducing the respective combinations of operators $\phi_{c, s}=\frac{1}{\sqrt{2}}\left(\phi_{\uparrow} \pm \phi_{\downarrow}\right)$, we can represent the low-energy Hamiltonian as $H=H_{c}+H_{s}$, where

$$
H_{c}=\int \frac{d x}{2 \pi}\left[u_{c} K_{c}\left(\pi \Pi_{c}\right)^{2}+\frac{u_{c}}{K_{c}}\left(\partial_{x} \phi_{c}\right)^{2}\right]
$$


describes the total density (or charge) fluctuations for incommensurate filling when umklapp terms are irrelevant, and

$$
H_{s}=\int \frac{d x}{2 \pi}\left[u_{s} K_{s}\left(\pi \Pi_{s}+\frac{\lambda}{a \sqrt{2}}\right)^{2}+\frac{u_{s}}{K_{s}}\left(\partial_{x} \phi_{s}\right)^{2}\right]-2 \Omega A_{0}^{2} \int d x \cos \sqrt{2} \theta_{s},
$$

describes the antisymmetric density (or spin) fluctuations. In Equations (2) and (3), $u_{s}$ and $u_{c}$ are, respectively, the velocity of antisymmetric and total density excitations, $A_{0}$ is a non-universal coefficient [21] while $K_{s}$ and $K_{c}$ are the corresponding Tomonaga-Luttinger (TL) exponents [38]. For two chains of hard core bosons, we have $u_{c}=u_{s}=2 t \sin \left(\pi \rho^{0} / 2\right)$ where $\rho^{0}$ is the average number of bosons per site and $K_{s}=K_{c}=1$.

The phase diagram of the Hamiltonian has been determined by looking at the behavior of the chiral current, i.e., the difference between the currents in the upper and lower leg, which is defined as

$$
\begin{aligned}
J_{s}(j, \lambda) & =-i t \sum_{\sigma} \sigma\left(b_{j, \sigma}^{\dagger} e^{i \lambda \sigma} b_{j+1, \sigma}-b_{j+1, \sigma}^{\dagger} e^{-i \lambda \sigma} b_{j, \sigma}\right), \\
& =\frac{u_{s} K_{s}}{\pi \sqrt{2}}\left(\partial_{x} \theta_{s}+\frac{\lambda}{a \sqrt{2}}\right) .
\end{aligned}
$$

As a function of the flux $\lambda$, the chiral current first increases linearly with $\lambda$ while being in the Meissner phase, and above a critical value of $\lambda$, it starts to decrease in the Vortex phase [2]. In this phase, the rung current starts to be different from zero. The phase diagram of the model can be obtained by analyzing the behavior of the rung and chiral current, and in Figure 1, the red-line is the phase boundary between the Vortex and the Meissner phase for the non-interacting case, while the black-line represents the phase boundary in the hard-core limit [20]. The major difference with respect to the non-interacting case is the persistence of the Meissner phase even for large values of the flux [18,19].

Beyond the chiral and rung current, the Meissner to Vortex phase transition can be traced out by looking at the behavior of the spectral function, which is more sensitive to incommensurations.

For the case of lattice bosons, the spectral function is defined as:

$$
A_{\sigma}(q, \omega)=i \sum_{j} \int d t \theta(t) e^{-i\left(q x_{j}-\omega t\right)}\left[\left\langle b_{j \sigma}(t) b_{0 \sigma}^{\dagger}(0)\right\rangle-\left\langle b_{0 \sigma}^{\dagger}(0) b_{j \sigma}(t)\right\rangle\right]
$$

where $x_{j}=j a$, $a$ being the lattice spacing, and can be experimentally accessed, for example, via radiofrequency (RF) spectroscopy techniques [30-32]. In the following, we will focus on the positive-frequency part of the spectral function, given by the first term in Equation (6). 


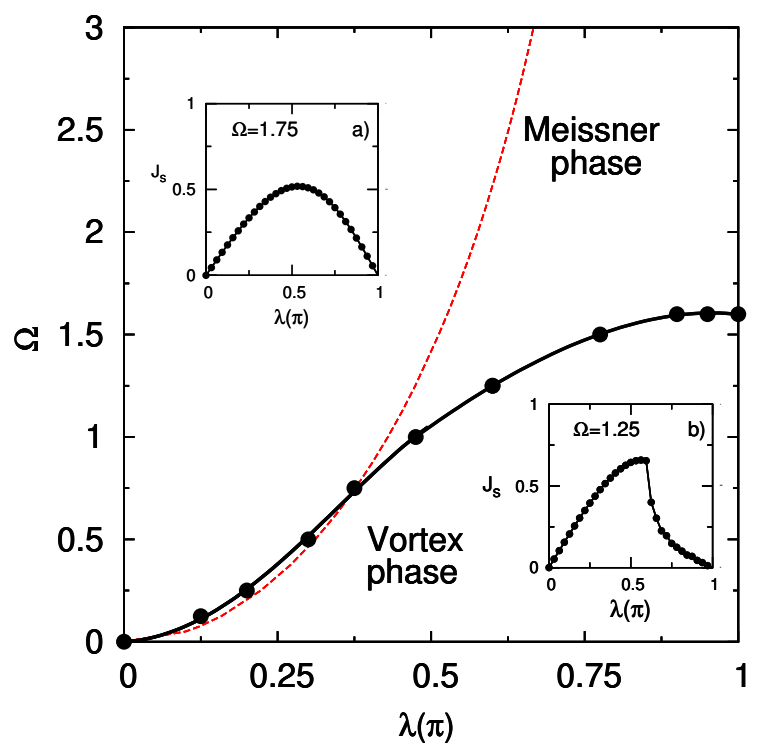

Figure 1. Phase diagram for a hard-core bosonic system on a ladder as a function of flux per plaquette $\lambda$ and $\Omega$, at the filling value $n=1$. The black solid line that joins solid dots is the phase boundary between the Meissner and the Vortex phase, while the dashed red line is the prediction for this boundary in the non-interacting system. In the insets, we show the different behavior of the spin-current $J_{s}(\lambda)$ for two values of interchain coupling $\Omega$ when there is the Meissner-Vortex transition and where there is not, respectively, panel (b) for $\Omega=1.25$ and (a) for $\Omega=1.75$ DMRG simulation results at $L=64$ in PBC.

\section{Spectral Function in the Meissner Phase for Weak Interchain Hopping}

Within the bosonization technique, the boson annihilation operator to the lowest order approximation can be represented as:

$$
\psi_{\sigma}(x, t)=b_{j, \sigma}(\tau) / \sqrt{a} \sim A_{0}\left\langle e^{i \frac{\theta_{s}}{\sqrt{2}}}\right\rangle e^{i \frac{\theta_{c}(j a, \tau)}{\sqrt{2}}} .
$$

$A_{0}$ is a non-universal constant and $\sigma$ stands for $\uparrow$ in the upper chain and $\downarrow$ for the lower chain. Knowing the Green's function for the field operator $\psi_{\sigma}$, one gets the spectral function as:

$$
A_{\sigma}(q, \omega) \sim A_{0}^{2}\left|\left\langle e^{i \frac{\theta_{s}}{\sqrt{2}}}\right\rangle\right|^{2} \int \frac{d x d t}{2 \pi} e^{-i(q x-\omega t)}\left(\frac{\alpha^{2}}{\left(\alpha-i u_{c} t\right)^{2}+x^{2}}\right)^{\frac{1}{8 K_{c}}},
$$

where $\alpha$ is the theory cutoff taken equal to the lattice spacing. The result of the integral yields

$$
A_{\sigma}(q, \omega) \sim \frac{\left(A_{0} \alpha\right)^{2} \pi}{u_{c}}\left|\left\langle e^{i \frac{\theta_{S}(x, t)}{\sqrt{2}}}\right\rangle\right|^{2} \frac{e^{2 \frac{\omega}{u_{c}} \alpha}}{\Gamma^{2}\left(\frac{1}{8 K_{c}}\right)}\left|\left(\frac{\omega^{2}}{u_{c}^{2}}-q^{2}\right) \alpha^{2}\right|^{\frac{1}{8 K_{c}}-1} \theta(\omega) \theta\left(\frac{|\omega|}{u_{c}}-|q|\right),
$$

The approximation of Equation (9) only yields the behavior of the spectral function at $\omega$ lower than the gap $\Delta_{S}$ in the $\theta_{S}$ modes. The actual correlation function can be obtained from the form factor expansion [39-43]. The lowest contribution from a soliton-antisoliton pair yields

$$
\left\langle T_{\tau} e^{i \frac{\theta_{s}(x, \tau)}{\sqrt{2}}} e^{-i \frac{\theta_{s}(0,0)}{\sqrt{2}}}\right\rangle=\left|\left\langle e^{i \frac{\theta_{s}(x, t)}{\sqrt{2}}}\right\rangle\right|^{2}+O\left(e^{-2 \Delta_{s} \sqrt{\left(x / u_{s}\right)^{2}+\tau^{2}}}\right),
$$


As a result, the Fourier transform of the full Matsubara Green's function is the sum of the contribution of Equation (9) and a second contribution analytic in a strip of the upper iv half plane of width proportional to the gap. This implies that the analytic continuation to real frequencies of this contribution is real until $\omega=2 \Delta_{s}$. There, a cut appears along the real frequency, and the imaginary part of that contribution to the Green's function becomes nonzero. This behavior is represented schematically in Figure 2. As the flux increases, the gap decreases linearly until it becomes zero at the commensurate-incommensurate point. At the point $K_{s}=1 / 2$, where the Hamiltonian $H_{s}$ can be fermionized, [20], the Matsubara Green's function can be calculated in terms of Ising order and disorder operators [44-48] correlation functions. If we look at the spectral function at $q= \pm 2 \pi \rho_{0}+\delta q$, with $\delta q \ll \rho_{0}$, the situation is simpler, and the correlation function to be calculated is

$$
A_{\uparrow}\left(2 \pi \rho_{0}+\delta q, \omega\right) \sim \int d x d t e^{-i(\delta q x-\omega t)}\left\langle e^{i\left(\frac{\theta_{c}}{\sqrt{2}}+\sqrt{2} \phi_{c}\right)(0,0)} e^{-i\left(\frac{\theta_{c}}{\sqrt{2}}+\sqrt{2} \phi_{c}\right)(x, t)}\right\rangle\left\langle\psi_{L}(0,0) \psi_{L}^{\dagger}(x, t)\right\rangle,
$$

thus, the spectral function is simply a convolution of the spectral function of a one-dimensional massive free Dirac fermion $\psi_{L}$ and the spectral function of a Tomonaga-Luttinger liquid. A schematic representation of the spectral function in the Meissner phase is presented in Figure 2.

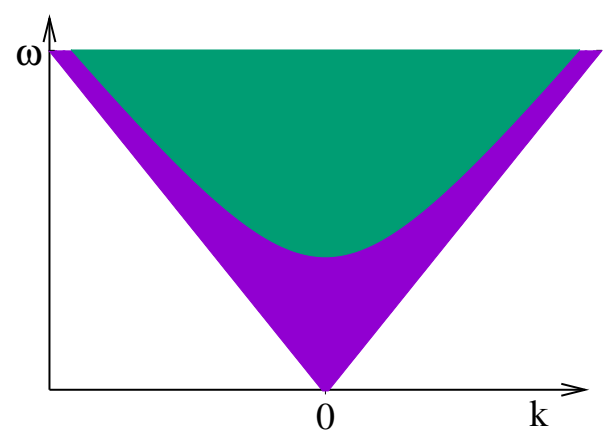

Figure 2. Schematic representation of the spectral function in the Meissner phase. The colored regions have a non-zero spectral weight. The violet region is the spectral weight coming only from the gapless charge modes, the spin modes remaining in their ground state. The green region represents the region where the gapped spin modes contribute to the spectral weight.

\section{Spectral Function in the Vortex Phase for Weak Interchain Hopping}

In the vortex phase, the boson field to the lowest order reads:

$$
\psi_{\sigma}(x, t)=b_{j, \sigma}(\tau) / \sqrt{a} \sim A_{0} e^{i \sigma q_{0}(\lambda) x_{j}} e^{i \frac{\theta_{s}(j a, \tau)}{\sqrt{2}}} e^{i \frac{\theta_{c}(j a, \tau)}{\sqrt{2}}},
$$

where $x_{j}=j a, q_{0}(\lambda)$ is the incommensurate wavevector of the vortex phase, given by $q_{0}(\lambda) \simeq \sqrt{\lambda^{2}-\lambda_{c}^{2}}$, where $\lambda_{c}$ is the critical spin-orbit coupling at which the degenerate minimum at $q=0$ in the single-particle spectrum splits into a double minima structure.

Thus, we find the Matsubara Green's function of the bosons in the form

$$
\left\langle T_{\tau} b_{j \sigma}(\tau) b_{0 \sigma}^{\dagger}(0)\right\rangle=A_{0}^{2} e^{-i \sigma q_{0}(\lambda) j a}\left(\frac{a^{2}}{(j a)^{2}+\left(u_{c} \tau\right)^{2}}\right)^{\frac{1}{8 K_{c}}}\left(\frac{a^{2}}{(j a)^{2}+\left(u_{s} \tau\right)^{2}}\right)^{\frac{1}{8 K_{s}^{*}}}
$$


and the spectral function is obtained by the integral:

$$
\begin{aligned}
A_{\sigma}(q, \omega) \sim A_{0}^{2} \int \frac{d x d t}{2 \pi} e^{-i(q x-\omega t)} & \left(\frac{\alpha}{\alpha-i\left(u_{c} t-x\right)}\right)^{\frac{1}{8 K_{c}}}\left(\frac{\alpha}{\alpha-i\left(u_{s} t-x\right)}\right)^{\frac{1}{8 K_{s}}} \\
& \times\left(\frac{\alpha}{\alpha-i\left(u_{c} t+x\right)}\right)^{\frac{1}{8 K_{c}}}\left(\frac{\alpha}{\alpha-i\left(u_{s} t+x\right)}\right)^{\frac{1}{8 K_{s}}},
\end{aligned}
$$

where $q_{0}(\lambda)$ is absorbed into $q$ and $K_{s} \rightarrow K_{s}^{\star}$. The Fourier transform of the Matsubara Green's function (13) can be calculated by the method outlined in [23,29], and after analytic continuation $i v \rightarrow \omega+i 0_{+}$it reads:

$$
\begin{aligned}
& G_{\sigma}(q, \omega)=f\left(K_{c}, K_{s}\right)\left|\omega^{2}-u_{s}^{2}\left(q+\sigma q_{0}(\lambda)\right)^{2}\right|^{\frac{1}{8 K_{c}}+\frac{1}{8 K_{s}^{*}}-1} e^{i \pi\left(1-\frac{1}{8 K_{c}}-\frac{1}{8 K_{s}^{*}}\right) \Theta\left(\omega^{2}-u_{s}^{2}\left(q+\sigma q_{0}(\lambda)\right)^{2}\right)} \\
& \times F_{1}\left(\frac{1}{8 K_{c}}, \frac{1}{8 K_{c}}+\frac{1}{8 K_{s}^{*}}-\frac{1}{2}, 1-\frac{1}{8 K_{c}}-\frac{1}{8 K_{s}^{*}}, \frac{1}{8 K_{c}}+\frac{1}{8 K_{s}^{*}} ; 1-\frac{u_{c}^{2}}{u_{s}^{2}}, 1-\frac{\omega^{2}-u_{c}^{2}\left(q+\sigma q_{0}(\lambda)\right)^{2}}{\omega^{2}-u_{s}^{2}\left(q+\sigma q_{0}(\lambda)\right)^{2}}\right),
\end{aligned}
$$

where $f\left(K_{c}, K_{s}\right)=\left(\frac{a}{2}\right)^{\frac{1}{4 K_{c}}+\frac{1}{4 K_{s}^{*}}} \frac{\Gamma\left(1-\frac{1}{8 K_{c}}-\frac{1}{8 K_{s}^{*}}\right)}{\Gamma\left(\frac{1}{8 K_{c}}+\frac{1}{8 K_{s}^{*}}\right)} u_{s}^{-\frac{1}{4 K_{c}}-\frac{1}{4 K_{s}^{*}}+1}$ and the function $F_{1}\left(a, b_{1}, b_{2}, c ; z_{1}, z_{2}\right)$ is an Appell hypergeometric function [49], which has a series representation in terms of two complex variables $z_{1}$ and $z_{2}$ when $\left|z_{1}\right|<1$ and $\left|z_{2}\right|<1$.

Singularities appear at $\omega_{1}^{\sigma}(q, \lambda)= \pm u_{c}\left(q+\sigma q_{0}(\lambda)\right)$ and at $\omega_{2}^{\sigma}(q, \lambda)= \pm u_{s}\left(q+\sigma q_{0}(\lambda)\right)$, and the power-law behavior of the spectral function near these points has been detailed in [29]. Some attention should be paid to extract the analytic continuation for values outside the radius of convergence of the Appell's function resorting to its integral representation possible when $\Re e[c-a]>0$, which in our case is always true by construction: $c-a=1 /\left(8 K_{s}^{*}\right)$. The behavior of the Green's function near the singularity points can be simplified as:

$$
\begin{aligned}
& G_{\sigma}(q, \omega) \simeq\left|\omega^{2}-\omega_{1}^{\sigma}(q, \lambda)^{2}\right|^{1 /\left(8 K_{c}\right)+1 /\left(4 K_{s}^{*}\right)-1} \\
& G_{\sigma}(q, \omega) \simeq\left|\omega^{2}-\omega_{2}^{\sigma}(q, \lambda)^{2}\right|^{1 /\left(8 K_{s}^{*}\right)+1 /\left(4 K_{c}\right)-1}
\end{aligned}
$$

In the Vortex phase, near the commensurate-incommensurate transition, the spin velocity $u_{s}^{*} \propto \sqrt{\lambda-\lambda_{c}}$, so we stay with the case where the charge velocity is larger than the spin one: in this case $1-u_{c}^{2} / u_{s}^{2} \leq 0$ and $\omega_{2}^{\sigma}(q, \lambda) \leq \omega_{1}^{\sigma}(q, \lambda)$. In the Vortex phase, when the hopping between the chains is not too large, $K_{s}^{*}>1 / 2$ and $K_{c}$ is near unity so that the imaginary part of the Green's function, i.e., the spectral function $A_{\sigma}(q, \omega)=-\Im G_{\sigma}(q, \omega) / \pi$, is divergent at the two poles $\omega_{1}$ and $\omega_{2}$ as shown in panel (a) of Figure 3. In order to wash out at least one of the divergencies near the two poles, small values of $K_{c}<1 / 2$ are required, signaling that density wave correlations are becoming important and eventually bringing a density-wave phase.

The behavior of the spin resolved spectral function $A_{\sigma}(q, \omega)$ for a fixed value of the applied flux is schematically shown in Figure 3 as a function of the $q$ and $\omega$ showing the contribution to spectral weight coming from the different singularities. 

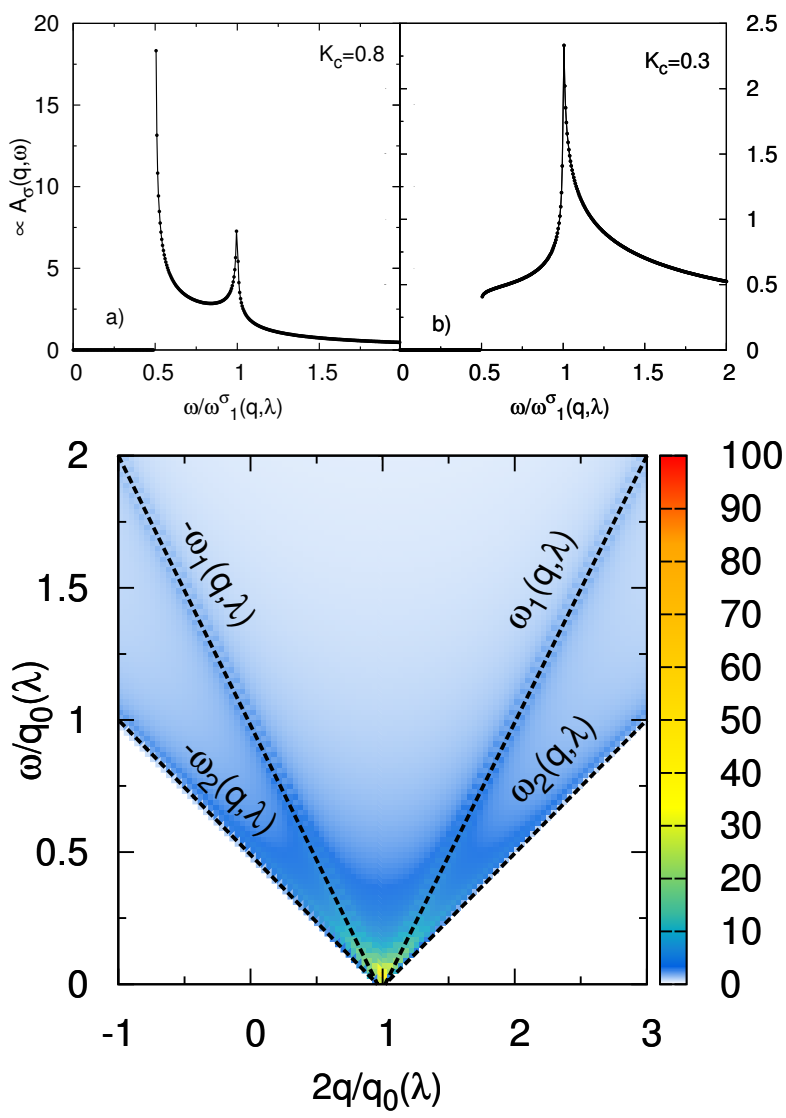

Figure 3. Spectral function $A_{\sigma}(q, \omega)$ as a function of $\omega / \omega_{1}^{\sigma}(q, \lambda)$ (on the top panels) for $u_{s}^{*} / u_{c}=0.5$ and $K_{s}^{*}=0.6$. In panel (a) we show the typical situation in the Vortex phase $\left(K_{c}=0.8\right)$, while in panel (b) we show the case $K_{c}=0.3$. In the lower panel: $A_{\sigma}(q, \omega)$ as a function of $\omega$ and $q$ for a fixed applied field $\lambda$ inducing a finite $q_{0}(\lambda)$ for $u_{s}^{*} / u_{c}=0.5$ with $K_{s}^{*}=K_{c}=0.6$. Finite spectral weights are present only in the colored region. The dashed blue lines correspond to $\omega_{1}(q, \lambda)$ and $\omega_{1}(q, \lambda)$

\section{Spectral Functions in the Weakly Interacting Regime From Bosonization}

We adopt here an alternative bosonization scheme [4], valid at weak interactions but arbitrary inter-leg tunnel coupling $\Omega$. In this regime, one can bosonize starting from the exact single-particle excitation spectrum [4], which displays a single minimum in the Meissner phase and two minima in the Vortex phase. In the Meissner state, the result of Equation (9) is recovered, but by construction of the bosonization scheme, the contribution of gapped modes at higher energy is not accessible. In the Vortex phase, at low energy, the field operators are approximated as [4]

$$
\begin{aligned}
& b_{j \uparrow}=u_{Q} \beta_{j+} e^{-i Q j}+v_{Q} \beta_{j-} e^{i Q j} \\
& b_{j \downarrow}=v_{Q} \beta_{j+} e^{-i Q j}+u_{Q} \beta_{j-} e^{i Q j}
\end{aligned}
$$

where $u_{Q}$ and $v_{Q}$ are the single-particle amplitudes, which diagonalize the non-interacting ladder Hamiltonian, calculated at the minima $\pm Q$ of the lower branch dispersion relation, and $\beta_{j \pm}=$ $\sum_{q} e^{-i q j a} \beta_{q \pm Q}$ with $\beta_{k}$ being the destruction operator of the lower single-particle excitation branch. Then, the field operators are bosonized as $\beta_{j \pm}=\sqrt{\bar{n}} e^{i \theta_{ \pm}\left(x_{j}\right)}$, and the Luttinger liquid Hamiltonian 
takes the usual quadratic form in the symmetric, antisymmetric sectors corresponding to the operators $\theta_{s(a)}=\left(\theta_{+} \pm \theta_{-}\right) / \sqrt{2}$. The associated Luttinger parameters are called $K_{s}, v_{s} K_{a}, v_{a}$.

The Green's function, calculated, for example, for the upper $\operatorname{leg} \sigma=1 / 2$, reads

$$
G_{\uparrow}(j, 0)=\left\langle b_{j \uparrow}(t) b_{0 \uparrow}^{\dagger}(0)\right\rangle=u_{Q}^{2}\left\langle\beta_{j+}(t) \beta_{0+}^{\dagger}(0)\right\rangle e^{-i Q j}+v_{Q}^{2}\left\langle\beta_{j-} \beta_{0-}^{\dagger}(0) e^{i Q j}\right.
$$

From bosonization, we obtain

$$
\left\langle\beta_{j \pm}(t) \beta_{0 \pm}^{\dagger}(0)\right\rangle=\bar{n}\left(\frac{a^{2}}{(j a)^{2}-\left(v_{s} t\right)^{2}}\right)^{1 /\left(8 K_{s}\right)}\left(\frac{a^{2}}{(j a)^{2}-\left(v_{a} t\right)^{2}}\right)^{1 /\left(8 K_{a}\right)}
$$

while $\left\langle\beta_{j \pm}(t) \beta_{0 \mp}^{+}(0)\right\rangle=0$. This can be Fourier transformed as done in Section 4 , yielding a spectral function with two incoherent contributions at $q= \pm Q$, each with a power-law singularity at the two excitation branches $\omega=v_{s, a}|q \pm Q|$. The same result is obtained in the lower leg, up to an exchange of $u_{Q}^{2}$ and $v_{Q}^{2}$.

\section{Spectral Function in the Bogoliubov Theory}

In the previous sections, we have derived the expressions for the spectral function with the bosonization technique, valid at intermediate and strong interactions. In the regime of very weak interactions and large filling of the lattice, a complementary approach is provided by the Bogoliubov theory [28]. The system is described by a two-component Bose-Einstein condensate with wavefunction $\Psi_{j \sigma}^{(0)}$ and small fluctuations on top of it. The condensate wavefunction $\Psi_{j \sigma}^{(0)}$ is obtained by solving the coupled discrete non-linear Schroedinger equations

$$
\begin{aligned}
\mu \Psi_{l, 1}^{(0)} & =-t \Psi_{l+1,1}^{(0)} e^{i \lambda}-t \Psi_{l-1,1}^{(0)} e^{-i \lambda} \\
& -(\Omega / 2) \Psi_{l, 2}^{(0)}+U\left|\Psi_{l, 1}^{(0)}\right|^{2} \Psi_{l, 1}^{(0)} \\
\mu \Psi_{l, 2}^{(0)} & =-t \Psi_{l+1,2}^{(0)} e^{-i \lambda}-t \Psi_{l-1,2}^{(0)} e^{i \lambda} \\
& -(\Omega / 2) \Psi_{l, 1}^{(0)}+U\left|\Psi_{l, 2}^{(0)}\right|^{2} \Psi_{l, 2}^{(0)},
\end{aligned}
$$

where $\mu$ is the chemical potential. The field operator is approximated by

$$
b_{j \sigma}(t) \simeq \Psi_{j \sigma}^{(0)}+\sum_{v} h_{v j}^{\sigma} \gamma_{v}-Q_{v j}^{\sigma *} \gamma_{v}^{\dagger}
$$

where $h_{v j}^{\sigma}$ and $Q_{v j}^{\sigma}$ are the Bogoliubov mode wavefunctions with energy $\omega_{v}$ and $\gamma_{v}$ are the quasiparticle creation and destruction field operators, satisfying bosonic commutation relations (see [28] for the full expressions).

Using Definition (6) for the spectral function, together with the mode expansion of the bosonic field operators of Equation (22), we obtain

$$
A_{\sigma}(q, \omega)_{\sigma}=\sum_{v}\left[\left|\tilde{h}_{v q}^{\sigma}\right|^{2} \delta\left(\omega-\omega_{v}\right)-\left|\tilde{Q}_{v q}^{\sigma}\right|^{2} \delta\left(\omega+\omega_{v}\right)\right]
$$

where $\tilde{h}_{v q}^{\sigma}=\sum_{j} e^{-i k a j} h_{v j}^{\sigma}$ and $\tilde{Q}_{v q}^{\sigma}=\sum_{j} e^{-i k a j} Q_{v j}^{\sigma}$.

The spectral function in the Bogoliubov approximation is illustrated in Figure 4. In the Meissner phase, a single branch in the spectrum is identified, corresponding to the Bogoliubov excitation mode, centered at $k=0$. Let us note that a gapped excitation branch of the Meissner phase is expected at energies 
of the order of $\Omega / t$, so well above the scale used in Figure 4. In the Vortex phase, we find two sound modes at low energy centered at $k= \pm q_{0}$, as well as weaker branches associated with the folding of the excitation spectrum in the presence of a modulated equilibrium density profile. The incommensurate wavevector $q_{0}$ corresponds to the non-zero momentum where an excitation may occur at zero energy, corresponding to the backscattering among the two minima of the single-particle dispersion, in Figure 4 it corresponds to the first replica of the excitation branch above $k=0$.

$A_{(1)}(q, \omega)$

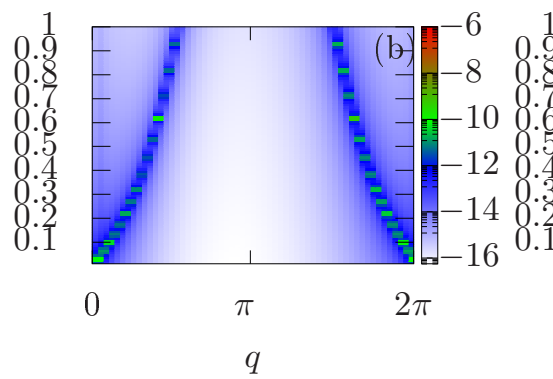

$A_{(1)}(q, \omega)$

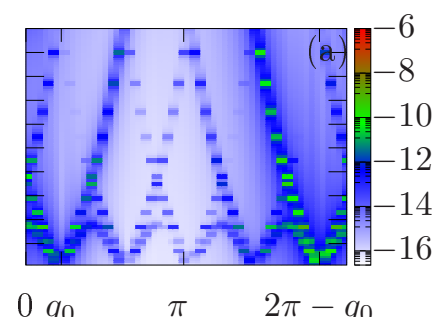

$q$

Figure 4. Spectral function $A_{\uparrow}(q, \omega)$ as a function of $\omega$ and $q$ for a fixed applied field $\lambda=\pi / 2$, in the Meissner phase (left panel, $\Omega / t=4$ ) and in the Vortex phase (right panel $\Omega / t=1.2$ ), for mean-field interaction parameter $U N /\left(N_{s} t\right)=0.6$ and number of sites $N_{S}=60$. Spectral lines are broadened by replacing a delta function with a Lorentzian (the width $10^{-14}$ in units of $\mathrm{J}$ is not visible on the scale of the figure, the broadening is mostly the pixelization of the image.

\section{Conclusions}

We have obtained the spectral functions of a two-leg boson ladder in an artificial gauge field. The bosonization approach, describing the regime of sufficiently strong interactions, predicts that in the Meissner phase, the low energy spectral weight is located near $\omega=0, q=0$. In the Vortex phase, it is located near $\omega=0, \pm q_{0}(\lambda)$. In both cases, the spectral weight is incoherent and characterized by power-law singularities at $\omega=u_{c}|q|$ (Meissner phase) or $\omega=u_{c}\left|q \pm q_{0}(\lambda)\right|$ (Vortex phase) with known exponents, and a specific incommensuration effect due to the shift of the spectral weight for $q \simeq q_{0}(\lambda) / 2$. In the Meissner phase, the gap in the antisymmetric density fluctuations translates as a power-law singularity of the spectral function at frequency $\omega>2 \Delta_{s}$. The Bogoliubov approximation, valid at weak interactions predicts delta-like spectral function, still keeping the main features: a single gapless excitation branch and a gapped one in the Meissner phase and two gapless excitation branches displaying incommensuration effects in the Vortex phase. The Bogoliubov theory misses the backscattering contributions to the spectral function, consistently with the bosonization predictions that their spectral weight is very small at weak interactions.

The present work could be extended in different directions. Exactly at the commensurate-incommensurate transition, the antisymmetric excitations are described by a gapless theory [50] with dynamical exponent $z=2$. The finite temperature correlation function has a known scaling form [51,52], and the spectral function at the commensurate-incommensurate transition can be obtained by convolution of that correlation function with the one of the charge modes. Such calculation is left for future work. Another possible extension is to consider the interleg interaction. Previous work has shown $[38,53]$ that it splits the commensurate-incommensurate transition point into an Ising transition point, a disorder point and a Berezinskii-Kosterltz-Thouless (BKT) transition point. An intermediate atomic density wave exists between the Ising and the BKT point, and it develops incommensuration at the disorder point. The atomic 
density wave could be characterized using the spectral functions as done in the present manuscript, both in its commensurate and in its incommensurate regime. A final possible extension is to consider the spectral functions in the presence of the second incommensuration [20,54] at $\lambda=\pi n$.

Author Contributions: Writing—original draft preparation, R.C.; writing—review and editing, S.D.P., A.M., E.O.; methodology, R.C., E.O., A.M.; software, S.D.P., N.V.; formal analysis, E.O. All authors have read and agreed to the published version of the manuscript.

Funding: The APC was funded by MDPI. ANR SuperRing project (Grant No.ANR-15-CE30-0012).

Acknowledgments: A.M. and N.V. acknowledge the ANR SuperRing project (Grant No. ANR-15-CE30-0012).

Conflicts of Interest: The authors declare no conflict of interest.

\section{References}

1. Kardar, M. Josephson-junction ladders and quantum fluctuations. Phys. Rev. B 1986, 33, 3125. [CrossRef] [PubMed]

2. Orignac, E.; Giamarchi, T. Meissner effect in a bosonic ladder. Phys. Rev. B 2001, 64, 144515. [CrossRef]

3. Cha, M.C.; Shin, J.G. Two peaks in the momentum distribution of bosons in a weakly frustrated two-leg optical ladder. Phys. Rev. A 2011, 83, 055602. [CrossRef]

4. Tokuno, A.; Georges, A. Ground States of a Bose-Hubbard Ladder in an Artificial Magnetic Field: Field-Theoretical Approach. New J. Phys. 2014, 16, 073005. [CrossRef]

5. Richaud, A.; Penna, V. Quantum dynamics of bosons in a two-ring ladder: Dynamical algebra, vortexlike excitations, and currents. Phys. Rev. A 2017, 96, 013620. [CrossRef]

6. Japaridze, G.I.; Nersesyan, A.A. Magnetic-field phase transition in a one-dimensional system of electrons with attraction. JETP Lett. 1978, 27, 334.

7. Pokrovsky, V.L.; Talapov, A.L. Ground State, Spectrum, and Phase Diagram of Two-Dimensional Incommensurate Crystals. Phys. Rev. Lett. 1979, 42, 65. [CrossRef]

8. Schulz, H.J. Critical behavior of commensurate-incommensurate phase transitions in two dimensions. Phys. Rev. B 1980, 22, 5274. [CrossRef]

9. Jaksch, D.; Zoller, P. The cold atom Hubbard toolbox. Ann. Phys. 2005, 315, 52. [CrossRef]

10. Lewenstein, M.; Sanpera, A.; Ahufinger, V.; Damski, B.; Sen De, A.; Sen, U. Ultracold atomic gases in optical lattices: mimicking condensed matter physics and beyond. Ann. Phys. 2007, 56, 243. [CrossRef]

11. Bloch, I.; Dalibard, J.; Zwerger, W. Many-body physics with ultracold gases. Rev. Mod. Phys. 2008, 80, 885. [CrossRef]

12. Dalibard, J.; Gerbier, F.; Juzeliūnas, G.; Öhberg, P. Colloquium: Artificial gauge potentials for neutral atoms. Rev. Mod. Phys. 2011, 83, 1523. [CrossRef]

13. Zhang, S.; Cole, W.S.; Paramekanti, A.; Trivedi, N. Spin-Orbit Coupling In Optical Lattices. In Annual Review of Cold Atoms and Molecules; Madison, K.W., Bongs, K., Carr, L.D., Rey, A.M., Zhai, H., Eds.; World Scientific: Singapore, 2015; Volume 3, Chapter 3, p. 135. [CrossRef]

14. Barbarino, S.; Taddia, L.; Rossini, D.; Mazza, L.; Fazio, R. Synthetic gauge fields in synthetic dimensions: interactions and chiral edge modes. New J. Phys. 2016, 18, 035010. [CrossRef]

15. Osterloh, K.; Baig, M.; Santos, L.; Zoller, P.; Lewenstein, M. Cold Atoms in Non-Abelian Gauge Potentials: From the Hofstadter "Moth" to Lattice Gauge Theory. Phys. Rev. Lett. 2005, 95, 010403. [CrossRef]

16. Ruseckas, J.; Juzeliūnas, G.; Öhberg, P.; Fleischhauer, M. Non-Abelian Gauge Potentials for Ultracold Atoms with Degenerate Dark States. Phys. Rev. Lett. 2005, 95, 010404. [CrossRef]

17. Lin, Y.; Jimenez-Garcia, K.; Spielman, I.B. Spin-orbit-coupled Bose-Einstein condensates. Nature 2011, 471, 83. [CrossRef]

18. Piraud, M.; Cai, Z.; McCulloch, I.P.; Schollwöck, U. Quantum magnetism of bosons with synthetic gauge fields in one-dimensional optical lattices: A Density Matrix Renormalization Group study. Phys. Rev. A 2014, 89, 063618. [CrossRef] 
19. Piraud, M.; Heidrich-Meisner, F.; McCulloch, I.P.; Greschner, S.; Vekua, T.; Schollwöck, U. Vortex and Meissner phases of strongly interacting bosons on a two-leg ladder. Phys. Rev. B 2015, 91, 140406. [CrossRef]

20. Orignac, E.; Citro, R.; Di Dio, M.; De Palo, S.; Chiofalo, M.L. Incommensurate phases of a bosonic two-leg ladder under a flux. New J. Phys. 2016, 18, 055017. [CrossRef]

21. Giamarchi, T. Quantum Physics in One Dimension; Oxford University Press: Oxford, UK, 2004.

22. Cazalilla, M.A.; Citro, R.; Giamarchi, T.; Orignac, E.; Rigol, M. One dimensional Bosons: From Condensed Matter Systems to Ultracold Gases. Rev. Mod. Phys. 2011, 83, 1405. [CrossRef]

23. Kleine, A.; Kollath, C.; McCulloch, I.; Giamarchi, T.; Schollwoeck, U. Spin-charge separation in two-component Bose gases. Phys. Rev. A 2008, 77, 013607. [CrossRef]

24. Dhar, A.; Maji, M.; Mishra, T.; Pai, R.V.; Mukerjee, S.; Paramekanti, A. Bose-Hubbard model in a strong effective magnetic field: Emergence of a chiral Mott insulator ground state. Phys. Rev. A 2012, 85, 041602. [CrossRef]

25. Dhar, A.; Mishra, T.; Maji, M.; Pai, R.V.; Mukerjee, S.; Paramekanti, A. Chiral Mott insulator with staggered loop currents in the fully frustrated Bose-Hubbard model. Phys. Rev. B 2013, 87, 174501. [CrossRef]

26. Petrescu, A.; Le Hur, K. Bosonic Mott Insulator with Meissner Currents. Phys. Rev. Lett. 2013, $111,150601$. [CrossRef] [PubMed]

27. Petrescu, A.; Le Hur, K. Chiral Mott insulators, Meissner effect, and Laughlin states in quantum ladders. Phys. Rev. B 2015, 91, 054520. [CrossRef]

28. Victorin, N.; Pedri, P.; Minguzzi, A. Excitation spectrum and supersolidity of a two-leg bosonic ring ladder. Phys. Rev. A 2020, arXiv:1910.06410.

29. Iucci, A.; Fiete, G.A.; Giamarchi, T. Fourier transform of the $2 k_{F}$ Luttinger liquid density correlation function with different spin and charge velocities. Phys. Rev. B 2007, 75, 205116. [CrossRef]

30. Fabbri, N.; Panfil, M.; Clément, D.; Fallani, L.; Inguscio, M.; Fort, C.; Caux, J.S. Dynamical structure factor of one-dimensional Bose gases: Experimental signatures of beyond-Luttinger-liquid physics. Phys. Rev. A 2015, 91, 043617. [CrossRef]

31. Dao, T.L.; Georges, A.; Dalibard, J.; Salomon, C.; Carusotto, I. Measuring the One-Particle Excitations of Ultracold Fermionic Atoms by Stimulated Raman Spectroscopy. Phys. Rev. Lett. 2007, 98, 240402. [CrossRef]

32. Stewart, J.T.; Gaebler, J.P.; Jin, D.S. Using photoemission spectroscopy to probe a strongly interacting Fermi gas. Nature 2008, 454, 744. [CrossRef]

33. Strinati, M.C.; Cornfeld, E.; Rossini, D.; Barbarino, S.; Dalmonte, M.; Fazio, R.; Sela, E.; Mazza, L. Laughlin-like states in bosonic and fermionic atomic synthetic ladders. Phys. Rev. X 2017, 7, 021033.

34. Celi, A.; Massignan, P.; Ruseckas, J.; Goldman, N.; Spielman, I.B.; Juzeliūnas, G.; Lewenstein, M. Synthetic Gauge Fields in Synthetic Dimensions. Phys. Rev. Lett. 2014, 112, 043001. [CrossRef] [PubMed]

35. Saito, T.Y.; Furukawa, S. Devil's staircases in synthetic dimensions and gauge fields. Phys. Rev. A 2017, 95, 043613. [CrossRef]

36. Livi, L.F.; Cappellini, G.; Diem, M.; Franchi, L.; Clivati, C.; Frittelli, M.; Levi, F.; Calonico, D.; Catani, J.; Inguscio, M.; et al. Synthetic Dimensions and Spin-Orbit Coupling with an Optical Clock Transition. Phys. Rev. Lett. 2016, 117, 220401. [CrossRef] [PubMed]

37. Haldane, F.D.M. Effective Harmonic-Fluid Approach to Low-Energy Properties of One-Dimensional Quantum Fluids. Phys. Rev. Lett. 1981, 47, 1840. [CrossRef]

38. Citro, R.; Palo, S.D.; Dio, M.D.; Orignac, E. Quantum phase transitions of a two-leg bosonic ladder in an artificial gauge field. Phys. Rev. B 2018, 97, 174523, [CrossRef]

39. Karowski, M.; Wiesz, P. Exact form factors in (1+1)Dimensional Field theoretic models with soliton behavior. Nucl. Phys. B 1978, 139, 455. [CrossRef]

40. Smirnov, F.A. Form Factors in Completely Integrable Models of Quantum Field Theory; World Scientific: Singapore, 1992.

41. Babujian, H.; Fring, A.; Karowski, M.; Zapletal, A. Exact Form Factors in Integrable Quantum Field Theories: the Sine-Gordon Model. Nucl. Phys. B 1999, 538, 535. [CrossRef]

42. Babujian, H.; Karowski, M. Exact form factors in integrable quantum field theories: the sine-Gordon model (II). Nucl. Phys. B 2002, 620, 407. [CrossRef] 
43. Essler, F.H.; Konik, R.M. Applications of Massive Integrable Quantum Field Theories to Problems in Condensed Matter Physics. From Fields Strings Circumnavigat. Theoret. Phys. 2005, 684-830._0020. [CrossRef]

44. José, J.V.; Kadanoff, L.P.; Kirkpatrick, S.; Nelson, D.R. Renormalization, vortices, and symmetry-breaking perturbations in the two-dimensional planar model. Phys. Rev. B 1977, 16, 1217. [CrossRef]

45. Zuber, J.B.; Itzykson, C. Quantum field theory and the two-dimensional Ising model. Phys. Rev. D 1977, 15, 2875. [CrossRef]

46. Schroer, B.; Truong, T.T. The order/disorder quantum field operators associated with the two-dimewnsional Ising model in the continuum limit. Nucl. Phys. B 1978, 144, 80. [CrossRef]

47. Ogilvie, M. Spin waves, vortices, Fermions and duality in the Ising and Baxter models. Ann. Phys. 1981, 136, 273. [CrossRef]

48. Boyanovsky, D. Field theory of the two-dimensional Ising model: Conformal invariance, order and disorder, and bosonization. Phys. Rev. B 1989, 39, 6744. [CrossRef] [PubMed]

49. Olver, F.; Lozier, D.; Boisvert, R.; Clark, C. (Eds.) NIST Handbook of Mathematical Functions; Cambridge University Press: Cambridge, UK, 2010.

50. Sachdev, S.; Senthil, T.; Shankar, R. Finite-temperature properties of quantum antiferromagnets in a uniform magnetic field in one and two dimensions. Phys. Rev. B 1994, 50, 258. [CrossRef]

51. Barthel, T.; Schollwöck, U.; Sachdev, S. Scaling of the thermal spectral function for quantum critical bosons in one dimension. arXiv 2012, arXiv:1212.3570.

52. Blosser, D.; Bhartiya, V.K.; Voneshen, D.J.; Zheludev, A. \$z=2\$ Quantum Critical Dynamics in a Spin Ladder. Phys. Rev. Lett. 2018, 121, 247201. [CrossRef]

53. Orignac, E.; Citro, R.; Di Dio, M.; De Palo, S. Vortex lattice melting in a boson-ladder in artificial gauge $\mathrm{f}$ ield. Phys. Rev. B 2017, 96, 014518. [CrossRef]

54. Di Dio, M.; De Palo, S.; Orignac, E.; Citro, R.; Chiofalo, M.L. Persisting Meissner state and incommensurate phases of hard-core boson ladders in a flux. Phys. Rev. B 2015, 92, 060506. [CrossRef]

(C) 2020 by the authors. Licensee MDPI, Basel, Switzerland. This article is an open access article distributed under the terms and conditions of the Creative Commons Attribution (CC BY) license (http:/ / creativecommons.org/licenses/by/4.0/). 